\title{
Not a Painless Condition: Rheumatological and Musculoskeletal Symptoms in Type 2 Diabetes, and the Implications for Exercise Participation
}

Emily R Cox ${ }^{1 *}$, Jeff S Coombes ${ }^{1}$, Shelley E Keating ${ }^{1}$, Nicola W Burton ${ }^{2}$ \& Brooke K Coombes $^{3}$

${ }^{1}$ School of Human Movement and Nutrition Sciences, The University of Queensland, St Lucia, Queensland, Australia

${ }^{2}$ School of Applied Psychology, Griffith University, Mt Gravatt, Queensland, Australia

${ }^{3}$ School of Allied Health Sciences, Griffith University, Nathan, Queensland, Australia

*Address correspondence and reprint requests to this author at the School of Human Movement and Nutrition Sciences, University of Queensland, St Lucia, Australia; Tel: +61 7 3365 6767; E-mail: emily.cox@uq.net.au 


\section{Abstract}

Objectives: People with type 2 diabetes (T2D) are more likely to develop a range of rheumatological and musculoskeletal symptoms (RMS), and experience both chronic and widespread pain, compared with the general population. However, these symptoms are not commonly acknowledged by researchers, which hampers our understanding of the impact on this population. Since exercise is a key lifestyle management strategy for T2D and participation levels are typically low, understanding the potential impact of RMS on exercise participation is critical. The aim of this review is to summarise the literature regarding the prevalence and pathophysiology of RMS in T2D, the evidence for the benefits and risks associated with exercise on RMS, and the currently available tools for the reporting of RMS in both research studies and community settings.

Methods: A narrative review.

Results: There are numerous exercise trials in T2D, but few have sufficiently reported pain-related adverse events and even fewer have investigated the effects of exercise on RMS and chronic pain. Discussion: Recommendations for future research are provided.

Key words: Type 2 diabetes, chronic pain, musculoskeletal symptoms, exercise, self-report tools

\subsection{Introduction}

The macrovascular and microvascular complications associated with type 2 diabetes mellitus (T2D) such as peripheral vascular disease, ischaemic heart disease, retinopathy and nephropathy, are considered the major cause of morbidity and mortality. Rheumatological and musculoskeletal symptoms (RMS) in these individuals are often overlooked, despite their ability to adversely impact quality of life, independence and exercise participation. RMS may present alone or alongside other diabetic complications such as diabetic symmetrical polyneuropathy (DSPN), although the impact of this clustering of comorbidities is poorly understood. The aims of this review are to summarise the literature regarding the pathophysiology and prevalence of RMS and chronic pain in people with T2D. Second, evidence for the effects of exercise on RMS, focussing on people with T2D, will be reviewed. 
Third, a review of currently available self-report tools to evaluate RMS and monitor the effects of exercise interventions on RMS, and recommendations for minimum reporting of information in clinical trials or community settings will be presented. There is much work to be done in this area, but it is hoped this review will draw greater attention to the burden of RMS and chronic pain in people with T2D and lead to more research to determine the benefits and risks of exercise training in these individuals.

\subsection{Impact of RMS and chronic pain on physical health}

Pain is "an unpleasant sensory or emotional experience associated with actual or potential tissue damage, or described in terms of such damage"[1]. Two thirds of Australians aged 15 years and over report experiencing bodily pain, with around 1 in 10 describing severe or very severe levels [2]. Further, chronic pain contributed $12 \%$ of the total burden of disease and injury in Australia in 2011, which is a total of 521,286 disability-adjusted life years [3]. Chronic musculoskeletal pain is highly prevalent in people with T2D [4-8]. Based on a survey of 951 people with T2D and 2,923 matched people without T2D from the Danish population, the prevalence of RMS was 1.7-2.1 times more frequent in people with T2D than in the general population $(p<.001)$ [9]. Another cross-sectional study, which aimed to determine the prevalence of pain and its association with glycaemic control and physical functioning in 11,689 people with $\mathrm{T} 2 \mathrm{D}$, found that $57.8 \%$ of participants had moderate to extreme pain [10].

Chronic pain is frequently associated with physical, psychological and social issues which may adversely impact a person's ability to perform activities of daily living (ADLs) and consequently quality of life [11-13]. This includes sleep disturbances, fatigue [14, 15], lack of independence [16, 17], and reduced work productivity $[18,19]$. For people with T2D, additional issues include poorer diabetes self-management, poor mental health, and an increase in the number of doctor visits [10, 20-22]. Of major importance, the presence of chronic pain significantly impacts an individual's exercise 
participation $[2,11,23]$, which is the key lifestyle strategy recommended for people with T2D for glycaemic control and reduction of weight $[20,23]$. In a general population based sample aged 45 years and over, adults with severe or very severe pain were more likely than those without pain to be overweight or obese ( $80 \%$ versus $67 \%$ ) and to lead an inactive lifestyle (53\% versus $37 \%)$ [2]. Avoidance of regular exercise may lead to a progressive decrease in strength and flexibility as well as an increase in body weight, which may exacerbate pain and disability [11].

\subsection{Prevalence and pathophysiology of RMS and chronic pain in people with T2D}

People with T2D have an increased risk of developing specific RMS such as adhesive capsulitis of the shoulder, tendinopathy, diffuse idiopathic skeletal hyperostosis (DISH), Dupuytren's disease and limited joint mobility syndrome (LMS) [24]. There are a number of other musculoskeletal conditions such as osteoarthritis (OA), low back pain (LBP), carpal tunnel syndrome and gout [25], which are also common in people with T2D, although a causal relationship has not been established $[24,26]$. It is believed that the high comorbidity may be a result of the shared risk factors between T2D and RMS, including obesity, hyperlipidaemia, inactive lifestyle, hypertension, chronic kidney disease, and older age $[27,28]$. Psychosocial issues such as depression [29, 30], fear of injury [31] and impaired quality of life [32] have also been linked with RMS and chronic pain in T2D.

Multiple mechanisms have been suggested to underlie the development and persistence of chronic RMS and pain in people with T2D. T2D is thought to increase the expression of pro-inflammatory cytokines [33] and proliferation of fibroblasts [34], both of which also play a key role in painful processes [35]. Animal studies have also shown altered mechanical properties of tendons in animals with chemically-induced diabetes mellitus [36, 37]. It is believed chronic hyperglycaemia, via abnormal glucose regulation, affects the collagen cross-linking in the connective tissue due to the accumulation of advanced glycation end products $[4,9,38,39]$. This may result in increased tissue stiffness, which may lead to microdamage, inflammation and inhibited soft tissue repair. Further, the high levels of 
insulin and insulin-like growth factor seen in T2D stimulates bone formation and results in abnormal collagen deposition in the connective tissues [40]. With regards to neural damage, it has been suggested that insulin resistance promotes the production of reactive oxygen species, resulting in endothelial dysfunction and ultimately neuro-ischemia [41]. Detailed reviews of these mechanisms are found elsewhere $[4,39,42]$.

\subsection{Adhesive Capsulitis}

Adhesive capsulitis, also known as frozen shoulder, is characterized by pain and increasing stiffness of the glenohumeral joint resulting in restricted movement, particularly with external rotation and abduction [43]. People with T2D are five times more likely (95\% $\mathrm{Cl} 3.2-7.7, \mathrm{p}<0.001)$ to develop adhesive capsulitis than non-T2D controls [43]. The estimated prevalence of adhesive capsulitis ranges from $10.5-31.8 \%$ in people with T2D compared with $2.5-10.3 \%$ in the general population $[44,45]$. Nearly one third of cases occur bilaterally, with unilateral cases affecting the dominant side more often $[45,46]$. Compared with the general population, adhesive capsulitis begins at an earlier age and is less painful in people with T2D, although it has a poorer prognosis and is less responsive to non-operative treatment [47].

\subsection{Tendinopathy}

A systematic review of 31 studies found that people with T2D are more than three times as likely (OR $3.67,95 \% \mathrm{Cl} 2.71-4.97)$ to develop both painful disorders of the tendon and asymptomatic structural tendon pathology than age and sex matched non-T2D controls [48]. Risk factors for the development of tendinopathy in T2D include the increased tendon thickness present in T2D, age and the length of time the patient has had T2D [48-50]. The exact cause is not clear due to other contributing factors common in T2D such as increased adiposity and medical therapy (e.g. statins) also having an effect $[51,52]$. 
In another large epidemiological study of 1,296 people with T2D and 5,159 age and sex matched nonT2D controls, a 44\% greater risk of tendon rupture requiring hospitalisation was found in people with T2D (incident rate ratio $1.44(95 \% \mathrm{Cl} 1.10-1.87 ; \mathrm{p}=0.005)$. The rotator cuff was the most common site for a tear. Independent risk factors for any tendon rupture were greater body mass index (BMI) and alcohol consumption. After adjusting for these factors, the risk due to T2D was estimated to be as high as $1.84[53]$.

\subsection{Diffuse Idiopathic Skeletal Hyperostosis (DISH)}

DISH has been found to occur in $12-49 \%$ of people with T2D, compared with $1.6-13 \%$ of the general population $[54,55]$. With a prominent effect on the thoracolumbar spine, DISH is characterized by calcification of spinal ligaments, generalized ossification of ligaments and tendons (particularly the skull, pelvis, patellae and calcaneus) and new bone formation [54, 55]. Clinical manifestations of DISH include thoracic, cervical and lumbar pain and decreased range of motion. In more severe cases, this can also include dysphagia and neurological abnormalities [56].

\subsection{Dupuytren's Contracture}

Dupuytren's contracture is defined as chronic and idiopathic thickening of the palmar aponeurosis which leads to various degrees of flexion deformities of the fingers. Mustafa et al. (2016) completed a cross-sectional study on 1,000 people with T2D, with $19 \%$ reporting a diagnosis of Dupuytren's contracture [57]. Another study found that $42 \%$ of 206 participants with T2D were suffering from Dupuytren's contracture compared with $29 \%$ of 203 age, gender and occupation-matched participants [58]. Age, duration of diabetes, female gender and microangiopathy significantly increase the likelihood of developing this condition [57-59]. In the general population, Dupuytren's contracture typically effects the fifth finger, whilst participants with T2D are more so affected in the ring and middle fingers $[60,61]$. 


\subsection{Limited Joint Mobility Syndrome (LMS)}

LJMS of the hand, also known as cheiroarthropathy is caused by thickening of the skin over the dorsum of the hands resulting in reduced flexion of the metacarpophalangeal and interphalangeal joints [27]. Increased glycosylation of collagen in the skin and periarticular tissues, increased collagen degradation, diabetic microangiopathy, increased duration of diabetes and DSPN have been associated with this condition $[62,63]$. Pal et al. (1986) examined 109 people with T2D and 75 nonT2D people, clinically diagnosing LJMS of the hand in 49\% of participants with insulin-dependent T2D $(p<0.01)$ and $52 \%$ of participants with non-insulin dependent T2D $(p<0.001)$, compared with $20 \%$ of non-T2D participants [63]. LMS can also effect the small joints in the foot, with long-term progression resulting in impairment of the shoulder, hip, ankle or spine [64].

\subsection{Widespread Chronic Pain}

People with T2D also have a greater risk of daily widespread chronic pain. In a community study of 480 adults aged 30-65 years, Mantyselka et al. (2008) reported $42 \%$ of people with T2D and chronic pain experienced pain daily or continuously for at least 3 months compared with $18 \%$ of people without T2D [4]. Moreover, 32\% of people with T2D reported pain at more than three bodily sites [65]. In another study with 100 participants with type 1 diabetes and T2D, higher $\mathrm{HbA}_{1 \mathrm{c}}$ levels were observed in people with widespread chronic pain than those with no chronic pain $(9.2 \pm 1.1 \%$ vs $6.4 \pm$ 1.5\%) [66].

\subsection{Low Back Pain}

LBP is a common RMS with $11.9 \pm 2.0 \%$ of people worldwide suffering from the condition [67]. A crosssectional analysis of 2,096 Spanish twins demonstrated that T2D was associated with a higher prevalence of severe LBP (adjusted OR 1.63; 95\% Cl 1.00 to 2.64) than those without the disease [68]. A significant association was also seen between LBP and the risk of diabetes (RR 1.30; $95 \% \mathrm{Cl} 1.09$ to $1.54, p=0.003)[69]$. 


\subsection{Osteoarthritis}

$\mathrm{OA}$ is the most frequent RMS experienced in both the general and T2D population, causing joint pain, stiffness and disability. It is characterized by loss of cartilage, bone hypertrophy, sclerosis and synovial inflammation [70]. A recent systematic review and meta-analysis of 10 studies and 16,742 patients concluded that T2D was significantly associated with the presence of symptomatic OA (OR 1.21; 95\% $\mathrm{Cl} 1.02-1.41)$ [71]. These odds were increased when controlling for weight and $\mathrm{BMI}$ (OR $1.25 ; 95 \% \mathrm{CI}$ 1.05-1.46). Another systematic review and meta-analysis of 49 studies showed a mean OA prevalence of $29.5 \pm 1.2 \%$ in people with T2D. The risk of OA was again greater in people with T2D than those without (OR 1.46; 95\% Cl 1.08-1.96, $p=0.01$ ) [72].

\subsection{Diabetic Symmetrical Polyneuropathy}

DSPN, which is characterized by progressive sensory loss, is one of the most common long-term complications of T2D, affecting $16.3-50 \%$ of people with the disease [25, 73]. Approximately 1 in 4 people with DSPN also experience chronic pain described as burning, tingling, electric, sharp, shooting, and lancinating pain in the extremities [73]. Symptoms of DSPN respond poorly to conventional analgesics. Painful DSPN is known to affect physical functioning and quality of life [73] and if inadequately treated, is associated with increased anxiety, depression, and sleep disturbance [25]. While attention to symptoms of DSPN is more commonly considered in research studies, it is often under-recognized that RMS and DSPN often coexist. In their study of 255 people with painful DSPN, Gore et al. (2006) found nearly two-thirds (63\%) had other chronic musculoskeletal pain conditions, with OA (34\%) and nociceptive LBP (27\%) being the most common. 1 in 4 people (26\%) also had other chronic neuropathic pain conditions; carpal tunnel syndrome $(14 \%)$ and LBP with neuropathic involvement (13\%) were the most common [25]. 


\subsection{Effect of exercise on RMS and chronic pain}

Clinical guidelines advise exercise and diet modification be used for the management of T2D before initiation of medication [74, 75]. The benefits of exercise for prevention of diabetes-related complications such as cardiovascular disease, neuropathy and nephropathy are well documented [7581]. Exercise improves insulin sensitivity and diabetes pathologies through a number of mechanisms including the activation of AMP-activated protein kinase (AMPK) [82]. In contrast to the large amounts of data showing the benefits of exercise on insulin sensitivity, there is little evidence on the specific effects of exercise on RMS or chronic pain in people with T2D, though pilot studies are promising [8385]. Exercise is thought to be beneficial in managing RMS and chronic pain in the general population by reducing associated symptoms such as pain, poor sleep and subsequent fatigue, reduced functional capacity, inflammation, and depression and anxiety [86]. In people with T2D, improvements in cardiorespiratory fitness, strength and flexibility were associated with lower symptoms and better function of the limbs and spine following exercise, compared to a control intervention [85].

A recent overview of Cochrane reviews by Geneen and colleagues (2017) concluded that the quality of evidence surrounding the impact of exercise for chronic pain is low, mainly due to small sample sizes and short planned follow-up periods [87]. Based on the heterogeneity of doses, intensities and modalities of exercise investigated in research studies, there are no specific guidelines for exercise and chronic pain [87], although the beneficial effects of exercise are proposed to be volume dependent [85]. Laboratory-based research in healthy [88] and older [89] adults has shown that individuals who self-reported greater levels of vigorous and total physical activity exhibited enhanced pain modulatory function [88]. Aerobic exercise, strength training, flexibility training and movement therapies have been most often recommended as non-pharmacological treatment of chronic pain [86]. 
Low to moderate intensity aerobic exercise (50-60\% maximum heart rate) has been demonstrated to improve chronic pain symptoms in meta-analyses of randomized controlled trials with people with fibromyalgia [90-92]. Another recent systematic review has shown that aerobic exercise may also reduce neuropathy-related symptoms in people with T2D, with minimal adverse events [93]. If tolerated, moderate to vigorous intensity aerobic exercise (60-80\% maximum heart rate), which is the current recommended intensity for the general population for improvements in cardiorespiratory fitness, body weight and general health $[94,95]$, may also be done. Reduction in weight from aerobic exercise has been shown to reduce the loading on joints and provide relief from osteoarthritic pain [96].

Strength training is also well supported as a safe and effective management strategy for chronic pain [97-99]. Strength training increases muscles mass and strength, which aids in maintaining physical function and preventing the development of other health conditions such as sarcopenia, osteoporosis, cardiovascular disease and T2D [94].

With the main aim of improving joint range of motion and decreasing stiffness, flexibility training may also provide benefits in people with T2D, with or without chronic pain [87]. Movement therapies such as Tai Chi and yoga have also been investigated for use in chronic pain populations. As these therapies are of low intensity and address pain and physical function, as well as balance, joint mobility and cognitions related to pain, they are safe and well tolerated [100-102].

While evidence suggests regular exercise can reduce chronic pain, acute increases in physical loading, such as when commencing an exercise regime, may aggravate musculoskeletal symptoms. Johansen et al (2017) randomized 98 people with T2D into standard care (individualized counselling and medical therapy) or a lifestyle intervention (standard care as well as 5-6 exercise sessions per week and dietary plans for weight loss). One in five people in the lifestyle intervention group reported RMS or 
discomfort resulting in an inability to exercise for 7 or more consecutive days [103]. The Italian Diabetes and Exercise Study, which was a randomized controlled trial investigating the efficacy of an intensive exercise intervention strategy in people with T2D ( $n=606)$, reported 34 exercise-related, musculoskeletal adverse events in the exercise group. This included rotator cuff tendinopathy, aggravation of LBP or OA of hip or knee joint, shin splints/lower limb pain, as well as other/generalized musculoskeletal discomfort [104]. Thirteen participants (4.3\%) dropped out of the exercise intervention group ( $n=303$ ) due to adverse events, though the nature of the events which lead to this was not stated. Praet et al (2008) compared 12-months of either brisk walking or a medical fitness programme in 92 people with T2D. Of the 40 participants (43\%) who were no longer participating at 12-months, 19 (48\%) reported orthopaedic-related comorbidities such as overuse injuries and/or subclinical OA of the lower extremities as their reason for dropping out [105]. Exercise training studies frequently exclude potential participants based on the presence of RMS [104, 106-109], hence estimates of adverse events related to exercise training may be underestimated.

\subsection{Evaluation of Rheumatological and Musculoskeletal Symptoms and Chronic Pain}

Systematic assessment of the risk versus benefit of exercise interventions on chronic pain in people with T2D is challenging for several reasons. Musculoskeletal symptoms are not commonly explored during exercise interventions. In the aforementioned Cochrane review, only $25 \%$ of the studies included actively reported adverse events [87]. Another systematic review of randomized controlled trials in people with T2D found only 1 of the 136 exercise trials evaluated pain at baseline, and no trials were designed to specifically target the chronic pain [110]. The Italian Diabetes and Exercise Study used tailored exercise programs to minimize the risk of injury and worsening of RMS so to improve exercise adherence [85]. A self-reported questionnaire was used at baseline to evaluate RMS, however this was not repeated following the 12-month intervention, although joint-specific symptoms and functional status of 5 main body areas were only evaluated in a smaller cohort of participants. From these they were able to determine correlations between symptom scores and functional status, and 
exercise volume. Unfortunately, each of the 5 questionnaires used contains up to 50 items each, which is a significant participant burden and is unlikely to be replicated in future exercise studies.

Our review of the literature highlights a lack of validated instruments exist to capture the range of symptoms experienced by people with $\mathrm{T} 2 \mathrm{D}$, as well as the multidimensional characteristics of pain in this population. For example, questionnaires such as the Nordic Musculoskeletal Injury Questionnaire address only the prevalence of symptoms in multiple areas of the body without providing information regarding the severity or interference with ADLs. Other questionnaires such as the Brief Pain Inventory (BPI) and the Medical Outcomes Study 12-item Short-Form Health Survey (SF-12), lack the capacity to identify widespread chronic pain, which is common in people with T2D. Several questionnaires, such as the Neuropathy Total Symptom Score-6 (NTSS-6), focus on symptoms of DSPN, but do not consider symptoms which may overlap with non-neuropathic musculoskeletal conditions. A combination of these tools may be needed to fully capture the burden of RMS and chronic pain. Development of a self-report questionnaire that considers chronic widespread pain, region-specific RMS and DSPN, and evaluates their impact on ADLs, in this population is required.

\subsection{Recommendations}

In order to better understand and address chronic pain in people with T2D, there needs to be an improvement in the associated monitoring and reporting of adverse events in exercise trials, particularly those related to the exacerbation of RMS. We strongly recommend, as a standard requirement, reporting both the prevalence of acute injury during exercise and the prevalence of musculoskeletal pain or discomfort resulting in an inability to exercise for 7 or more consecutive days, as documented in a recent trial published in the Journal of the American Medical Association [103]. The combination of these two outcomes provides meaningful information about the impact of RMS and pain on exercise adherence and may provide insight into potential barriers of regular exercise in T2D populations. To improve the generalisability of study findings, we also recommend more explicit 
description of participant eligibility and reasons for exclusion due to pre-existing musculoskeletal conditions and chronic pain. To determine the efficacy of exercise training on chronic pain, future studies need to evaluate the presence, severity and interference of RMS and chronic pain at baseline and at follow up in both intervention and control or usual care groups.



Figure 1: Summary of Evidence Regarding Rheumatological and Musculoskeletal Symptoms in Type 2 Diabetes, and the Implications for Exercise Participation and Research

\subsection{Conclusion}

RMS and chronic pain are highly prevalent in people with T2D, inferring that T2D is not a painless condition. The impact of RMS and chronic pain on exercise, a key component of managing T2D, is not clearly understood, potentially hampering critical efforts to improve participation in this population. High quality research investigating the efficacy of exercise to improve RMS and chronic pain in people with T2D is urgently needed. There also needs to be an improvement in the reporting and monitoring of adverse events during exercise training studies. Greater attention toward RMS may help 
researchers and practitioners to understand, prevent and treat RMS and chronic pain and ultimately improve exercise adherence in people with T2D.

\section{Acknowledgements}

No sources of funding were used to assist in the preparation of this article.

\section{Conflicts of Interest}

The authors declare that they have no conflicts of interest, financial or otherwise, relevant to the views shared in this article. 


\section{References}

[1] Merskey H. The definition of pain. Eur Psychiatry 1991; 6(4): 153-9.

[2] Australian Bureau of Statistics. Characteristics of bodily pain in Australia [Online]. Canberra: ABS, 2012 [cited 16 Nov 2017]. ABS Cat. No. 4841.0. Available from: http://www.abs.gov.au.

[3] Australian Institute of Health and Welfare. The burden of musculoskeletal conditions in Austraila: a detailed analysis of the Australian Burden of Disease Study 2011. Australian Burden of Disease Study series no. 13. BOD 14, Canberra: AlHW; 2017.

[4] Mäntyselkä P, Miettola J, Niskanen L, Kumpusalo E. Chronic pain, impaired glucose tolerance and diabetes: a community-based study. Pain 2008; 137(1): 34-40.

[5] Hoff OM, Midthjell K, Zwart J-A, Hagen K. The association between diabetes mellitus, glucose, and chronic musculoskeletal complaints. Results from the Nord-Trøndelag Health Study. BMC Musculoskeletal Disorders 2008; 9(1): 160.

[6] Centres for Disease Control and Prevention. Arthritis as a potential barrier to physical activity among adults with diabetes--United States, 2005 and 2007. MMWR 2008; 57(18): 486-9.

[7] Wolak T, Weitzman S, Harman-Boehm I, Friger M, Sukenik S. Prevalence of fibromyalgia in type 2 diabetes mellitus. Harefuah 2001; 140(11): 1006-9, 120, 119.

[8] Abaraogu UO, Ochi C, Umahi E, Ogbonnaya C, Onah I. Individuals with type 2 diabetes are at higher risk of chronic musculoskeletal pain: a study with diabetes cohort. Int J Diabetes Dec Ctries 2017; 37(3): 267-71.

[9] Molsted S, Tribler J, Snorgaard O. Musculoskeletal pain in patients with type 2 diabetes. Diabetes Res Clin Pract 2012; 96(2): 135-40.

[10] Bair MJ, Brizendine EJ, Ackermann RT et al. Prevalence of pain and association with quality of life, depression and glycaemic control in patients with diabetes. Diabet Med 2010; 27(5): 578-84.

[11] Tüzün EH. Quality of life in chronic musculoskeletal pain. Best Pract Res Clin Rheumatol 2007; 21(3): 567-79.

[12] World Health Organization. The burden of musculoskeletal conditions at the start of the new millenium: report of a WHO scientific group. WHO Technical Report Series, 919; 2003.

[13] Dueñas M, Ojeda B, Salazar A, Mico JA, Failde I. A review of chronic pain impact on patients, their social environment and the health care system. J Pain Res 2016; 9: 457-67.

[14] O'Brien EM, Waxenberg LB, Atchison JW et al. Intraindividual variability in daily sleep and pain ratings among chronic pain patients: bidirectional association and the role of negative mood. Clin $J$ Pain 2011; 27(5): 425-33.

[15] Fishbain DA, Cole B, Cutler RB et al. Is pain fatiguing? A structured evidence-based review. Pain Med 2003; 4(1): 51-62.

[16] Jones J, Rutledge DN, Jones KD, Matallana L, Rooks DS. Self-assessed physical function levels of women with fibromyalgia: a national survey. Womens Health Issues 2008; 18(5): 406-12.

[17] Amris K, Waehrens EE, Jespersen A, Bliddal H, Danneskiold-Samsoe B. Observation-based assessment of functional ability in patients with chronic widespread pain: a cross-sectional study. Pain 2011; 152(11): 2470-6.

[18] Patel AS, Farquharson R, Carroll D et al. The impact and burden of chronic pain in the workplace: a qualitative systematic review. Pain Pract 2012; 12(7): 578-89.

[19] Stewart WF, Ricci JA, Chee E, Morganstein D, Lipton R. Lost productive time and cost due to common pain conditions in the US workforce. JAMA 2003; 290(18): 2443-54.

[20] Krein SL, Heisler M, Piette JD, Makki F, Kerr EA. The Effect of Chronic Pain on Diabetes Patients' Self-Management. Diabetes Care 2005; 28(1): 65.

[21] Pai L-W, Hung C-T, Li S-F et al. Musculoskeletal pain in people with and without type 2 diabetes in Taiwan: a population-based, retrospective cohort study. BMC Musculoskeletal Disorders 2015; 16(1): 364. 
[22] van der Zee-Neuen A, Putrik P, Ramiro S et al. Impact of Chronic Diseases and Multimorbidity on Health and Health Care Costs: The Additional Role of Musculoskeletal Disorders. Arthritis Care Res (Hoboken) 2016; 68(12): 1823-31.

[23] McBeth J, Nicholl BI, Cordingley L, Davies KA, Macfarlane GJ. Chronic widespread pain predicts physical inactivity: results from the prospective EPIFUND study. Eur J Pain 2010; 14(9): 972-9.

[24] Al-Homood IA. Rheumatic conditions in patients with diabetes mellitus. Clin Rheumatol 2013; 32(5): 527-33.

[25] Gore M, Brandenburg NA, Hoffman DL, Tai KS, Stacey B. Burden of illness in painful diabetic peripheral neuropathy: the patients' perspectives. J Pain 2006; 7(12): 892-900.

[26] Crispin JC, Alcocer-Varela J. Rheumatologic manifestations of diabetes mellitus. Am J Med 2003; 114(9): 753-7.

[27] Cagliero E, Apruzzese W, Perlmutter GS, Nathan DM. Musculoskeletal disorders of the hand and shoulder in patients with diabetes mellitus. Am J Med 2002; 112(6): 487-90.

[28] Scott A, Zwerver J, Grewal N et al. Lipids, adiposity and tendinopathy: is there a mechanistic link? Critical review. Br J Sports Med 2015; 49(15): 984.

[29] de Groot M, Anderson R, Freedland KE, Clouse RE, Lustman PJ. Association of depression and diabetes complications: a meta-analysis. Psychosomatic medicine 2001; 63(4): 619-30.

[30] Ludman EJ, Katon W, Russo J et al. Depression and diabetes symptom burden. General Hospital Psychiatry 2004; 26(6): 430-6.

[31] Huebschmann AG, Crane LA, Belansky ES et al. Fear of Injury With Physical Activity Is Greater in Adults With Diabetes Than in Adults Without Diabetes. Diabetes Care 2011; 34(8): 1717.

[32] Benbow SJ, Wallymahmed ME, MacFarlane IA. Diabetic peripheral neuropathy and quality of life. QJM: An International Journal of Medicine 1998; 91(11): 733-7.

[33] Rodeo SA, Hannafin JA, Tom J, Warren RF, Wickiewicz TL. Immunolocalization of cytokines and their receptors in adhesive capsulitis of the shoulder. J Orthop Res 1997; 15(3): 427-36.

[34] Bunker TD, Anthony PP. The pathology of frozen shoulder. A Dupuytren-like disease. J Bone Joint Surg Br 1995; 77(5): 677-83.

[35] Calixto JB, Medeiros R, Fernandes ES et al. Kinin B1 receptors: key G-protein-coupled receptors and their role in inflammatory and painful processes. Br J Pharmacol 2004; 143(7): 803-18.

[36] de Oliveira RR, de Lira KD, Silveira PV et al. Mechanical properties of achilles tendon in rats induced to experimental diabetes. Ann Biomed Eng 2011; 39(5): 1528-34.

[37] Guney A, Vatansever F, Karaman I et al. Biomechanical properties of Achilles tendon in diabetic vs. non-diabetic patients. Exp Clin Endocrinol Diabetes 2015; 123(7): 428-32.

[38] Abate M, Schiavone C, Pelotti P, Salini V. Limited joint mobility (LM) in elderly subjects with type II diabetes mellitus. Arch Gerontol Geriatr 2011; 53(2): 135-40.

[39] Snedeker JG, Gautieri A. The role of collagen crosslinks in ageing and diabetes - the good, the bad, and the ugly. Muscles Ligaments Tendons J 2014; 4(3): 303-8.

[40] Kiss C, Szilágyi M, Paksy A, Poór G. Risk factors for diffuse idiopathic skeletal hyperostosis: a case-control study. Rheumatology (Oxford) 2002; 41(1): 27-30.

[41] Van Acker K, Bouhassira D, De Bacquer D et al. Prevalence and impact on quality of life of peripheral neuropathy with or without neuropathic pain in type 1 and type 2 diabetic patients attending hospital outpatients clinics. Diabetes Metab 2009; 35(3): 206-13.

[42] Lebiedz-Odrobina D, Kay J. Rheumatic manifestations of diabetes mellitus. Rheum Dis Clin North Am 2010; 36(4): 681-99.

[43] Zreik NH, Malik RA, Charalambous CP. Adhesive capsulitis of the shoulder and diabetes: a metaanalysis of prevalence. Muscles Ligaments Tendons J 2016; 6(1): 26-34.

[44] Bridgman JF. Periarthritis of the shoulder and diabetes mellitus. Ann Rheum Dis 1972; 31(1): 6971.

[45] Mavrikakis ME, Drimis S, Kontoyannis DA et al. Calcific shoulder periarthritis (tendinitis) in adult onset diabetes mellitus: a controlled study. Ann Rheum Dis 1989; 48(3): 211-4. 
[46] Balci N, Balci MK, Tüzüner S. Shoulder Adhesive Capsulitis and Shoulder Range of Motion in Type II Diabetes Mellitus: Association with Diabetic Complications. J Diabetes Complications 1999; 13(3): 135-40.

[47] Smith L, Burnet S, McNeil J. Musculoskeletal manifestations of diabetes mellitus. Br J Sports Med 2003; 37(1): 30-5.

[48] Ranger TA, Wong AMY, Cook JL, Gaida JE. Is there an association between tendinopathy and diabetes mellitus? A systematic review with meta-analysis. Br J Sports Med 2016; 50(16): 982-9. [49] Akturk M, Karaahmetoglu S, Kacar M, Muftuoglu O. Thickness of the Supraspinatus and Biceps Tendons in Diabetic Patients. Diabetes Care 2002; 25(2): 408.

[50] Akturk M, Ozdemir A, Maral I, Yetkin I, Arslan M. Evaluation of Achilles Tendon Thickening in Type 2 Diabetes Mellitus. Exp Clin Endocrinol Diabetes 2007; 115(02): 92-6.

[51] Marie I, Delafenetre H, Massy N, Thuillez C, Noblet C. Tendinous disorders attributed to statins: a study on ninety-six spontaneous reports in the period 1990-2005 and review of the literature. Arthritis Rheum 2008; 59(3): 367-72.

[52] Franceschi F, Papalia R, Paciotti M et al. Obesity as a risk factor for tendinopathy: a systematic review. Int J Endocrinol 2014; 2014: 670262.

[53] Zakaria MH, Davis WA, Davis TM. Incidence and predictors of hospitalization for tendon rupture in type 2 diabetes: the Fremantle diabetes study. Diabet Med 2014; 31(4): 425-30.

[54] Sencan D, Elden H, Nacitarhan V, Sencan M, Kaptanoglu E. The prevalence of diffuse idiopathic skeletal hyperostosis in patients with diabetes mellitus. Rheumatol Int 2005; 25(7): 518-21.

[55] Belanger TA, Rowe DE. Diffuse idiopathic skeletal hyperostosis: musculoskeletal manifestations. J Am Acad Orthop Surg 2001; 9(4): 258-67.

[56] Cammisa M, De Serio A, Guglielmi G. Diffuse idiopathic skeletal hyperostosis. Eur J Radiol 1998; 27, Supplement 1: S7-S11.

[57] Mustafa KN, Khader YS, Bsoul AK, Ajlouni K. Musculoskeletal disorders of the hand in type 2 diabetes mellitus: prevalence and its associated factors. Int J Rheum Dis 2016; 19(7): 730-5.

[58] Ravindran Rajendran S, Bhansali A, Walia R et al. Prevalence and pattern of hand soft-tissue changes in type 2 diabetes mellitus. Diabetes Metab 2011; 37(4): 312-7.

[59] Arkkila PE, Gautier J-F. Musculoskeletal disorders in diabetes mellitus: an update. Best Pract Res Clin Rheumatol 2003; 17(6): 945-70.

[60] Noble J, Heathcote J, Cohen H. Diabetes mellitus in the aetiology of Dupuytren's disease. J Bone Joint Surg Br 1984; 66(3): 322-5.

[61] Kameyama M, Meguro SHU, Funae O, Atsumi Y, Ikegami H. The Presence of Limited Joint Mobility Is Significantly Associated with Multiple Digit Involvement by Stenosing Flexor Tenosynovitis in Diabetics. J Rheumatol 2009; 36(8): 1686.

[62] Wyatt LH, Ferrance RJ. The musculoskeletal effects of diabetes mellitus. J Can Chiropr Assoc 2006; 50(1): 43-50.

[63] Pal B, Anderson J, Dick WC, Griffiths ID. Limitation of joint mobility and shoulder capsulitis in insulin- and non-insulin-dependent diabetes mellitus. Br J Rheumatol 1986; 25(2): 147-51.

[64] Gerrits EG, Landman GW, Nijenhuis-Rosien L, Bilo HJ. Limited joint mobility syndrome in diabetes mellitus: A minireview. World J Diabetes 2015; 6(9): 1108-12.

[65] Mäntyselkä P, Miettola J, Niskanen L, Kumpusalo E. Persistent pain at multiple sitesConnection to glucose derangement. Diabetes Res Clin Pract 2009; 84(2): e30-e2.

[66] Tishler M, Smorodin T, Vazina-Amit M et al. Fibromyalgia in diabetes mellitus. Rheumatol Int 2003; 23(4): 171-3.

[67] Hoy D, Bain C, Williams $G$ et al. A systematic review of the global prevalence of low back pain. Arthritis Rheum 2012; 64(6): 2028-37.

[68] Dario A, Ferreira M, Refshauge $K$ et al. Mapping the association between back pain and type 2 diabetes: A cross-sectional and longitudinal study of adult Spanish twins. PLoS One 2017; 12(4): e0174757. 
[69] Heuch I, Heuch I, Hagen K et al. Is chronic low back pain a risk factor for diabetes? The NordTrøndelag Health Study. BMJ Open Diabetes Research and Care 2018; 6(1): e000569.

[70] Courties A, Sellam J. Osteoarthritis and type 2 diabetes mellitus: What are the links? Diabetes Res Clin Pract 2016; 122: 198-206.

[71] Williams MF, London DA, Husni EM, Navaneethan S, Kashyap SR. Type 2 diabetes and osteoarthritis: a systematic review and meta-analysis. J Diabetes Complications 2016; 30(5): 944-50.

[72] Louati K, Vidal C, Berenbaum F, Sellam J. Association between diabetes mellitus and osteoarthritis: systematic literature review and meta-analysis. RMD Open 2015; 1(1): e000077. [73] Davies M, Brophy S, Williams R, Taylor A. The prevalence, severity, and impact of painful diabetic peripheral neuropathy in type 2 diabetes. Diabetes Care 2006; 29(7): 1518-22.

[74] Kirwan JP, Sacks J, Nieuwoudt S. The essential role of exercise in the management of type 2 diabetes. Cleve Clin J Med 2017; 84(7 Suppl 1): S15-s21.

[75] Colberg SR, Sigal RJ, Fernhall B et al. Exercise and Type 2 Diabetes: The American College of Sports Medicine and the American Diabetes Association: joint position statement. Diabetes Care 2010; 33(12): e147-e67.

[76] Thomas DE, Elliott EJ, Naughton GA. Exercise for type 2 diabetes mellitus. Cochrane Database Syst Rev 2006(3): CD002968.

[77] Boulé NG, Haddad E, Kenny GP, Wells GA, Sigal RJ. Effects of exercise on glycemic control and body mass in type 2 diabetes mellitus: A meta-analysis of controlled clinical trials. JAMA 2001; 286(10): 1218-27.

[78] Boule NG, Kenny GP, Haddad E, Wells GA, Sigal RJ. Meta-analysis of the effect of structured exercise training on cardiorespiratory fitness in type 2 diabetes mellitus. Diabetologia 2003; 46(8): 1071-81.

[79] Balducci S, lacobellis G, Parisi L et al. Exercise training can modify the natural history of diabetic peripheral neuropathy. J Diabetes Complications 2006; 20(4): 216-23.

[80] Zoppini G, Targher G, Zamboni C et al. Effects of moderate-intensity exercise training on plasma biomarkers of inflammation and endothelial dysfunction in older patients with type 2 diabetes. Nutr Metab Cardiovasc Dis 2006; 16(8): 543-9.

[81] Loimaala A, Huikuri HV, Koobi T et al. Exercise training improves baroreflex sensitivity in type 2 diabetes. Diabetes 2003; 52(7): 1837-42.

[82] Finelli C, Tarantino G. Have guidelines addressing physical activity been established in nonalcoholic fatty liver disease? World J Gastroenterol 2012; 18(46): 6790-800.

[83] Kluding PM, Pasnoor M, Singh R et al. The effect of exercise on neuropathic symptoms, nerve function, and cutaneous innervation in people with diabetic peripheral neuropathy. $J$ Diabetes Complications 2012; 26(5): 424-9.

[84] Yoo M, D'Silva L, Martin K et al. Pilot Study of Exercise Therapy on Painful Diabetic Peripheral Neuropathy. Pain Med 2015; 16(8): 1482-9.

[85] Balducci S, Vulpiani MC, Pugliese L et al. Effect of supervised exercise training on musculoskeletal symptoms and function in patients with type 2 diabetes: the Italian Diabetes Exercise Study (IDES). Acta Diabetol 2014; 51(4): 647-54.

[86] Ambrose KR, Golightly YM. Physical exercise as non-pharmacological treatment of chronic pain: Why and when. Best Pract Res Clin Rheumatol 2015; 29(1): 120-30.

[87] Geneen L, Moore RA, Clarke C et al. Physical activity and exercise for chronic pain in adults: an overview of Cochrane Reviews. Cochrane Database Syst Rev 2017(4): CD011279.

[88] Naugle KM, Riley JL, 3rd. Self-reported physical activity predicts pain inhibitory and facilitatory function. Med Sci Sports Exerc 2014; 46(3): 622-9.

[89] Naugle KM, Ohlman T, Naugle KE, Riley ZA, Keith NR. Physical activity behavior predicts endogenous pain modulation in older adults. Pain 2017; 158(3): 383-90.

[90] Hauser W, Klose P, Langhorst J et al. Efficacy of different types of aerobic exercise in fibromyalgia syndrome: a systematic review and meta-analysis of randomised controlled trials.

Arthritis Res Ther 2010; 12(3): R79. 
[91] Jones KD, Adams D, Winters-Stone K, Burckhardt CS. A comprehensive review of 46 exercise treatment studies in fibromyalgia (1988-2005). Health Qual Life Outcomes 2006; 4: 67.

[92] Busch AJ, Barber KAR, Overend TJ, Peloso PMJ, Schachter CL. Exercise for treating fibromyalgia syndrome. Cochrane Database Syst Rev 2007(4): CD003786.

[93] Gu Y, Dennis SM, Kiernan MC, Harmer AR. Aerobic exercise training may improve nerve function in type 2 diabetes and pre-diabetes: A systematic review. Diabetes/metabolism research and reviews 2019; 35(2): e3099.

[94] Pescatello LS, Arena R, Riebe D, Thompson PD. ACSM's guidelines for exercise testing and prescription. Medicine ACoS, Ed. 9th ed. Philadelphia, PA: Wolters Kluwer/Lippincott Williams \& Wilkins 2014. pp. 456.

[95] Brown WJ, Bauman AE, Bull FC, Burton NW. Development of evidence-based physical activity recommendations for adults (18-64 years): report prepared for the Australian Government Department of Health, August 2012. 2013. Report No.: 1741860695.

[96] Messier SP, Gutekunst DJ, Davis C, DeVita P. Weight loss reduces knee-joint loads in overweight and obese older adults with knee osteoarthritis. Arthritis Rheum 2005; 52(7): 2026-32.

[97] Baillet A, Vaillant M, Guinot M, Juvin R, Gaudin P. Efficacy of resistance exercises in rheumatoid arthritis: meta-analysis of randomized controlled trials. Rheumatology (Oxford) 2012; 51(3): 519-27.

[98] Gavi MB, Vassalo DV, Amaral FT et al. Strengthening exercises improve symptoms and quality of life but do not change autonomic modulation in fibromyalgia: a randomized clinical trial. PLoS One 2014; 9(3): e90767.

[99] Busch AJ, Webber SC, Richards RS et al. Resistance exercise training for fibromyalgia. Cochrane Database Syst Rev 2013(12): Cd010884.

[100] Jones KD, Sherman CA, Mist SD et al. A randomized controlled trial of 8-form Tai chi improves symptoms and functional mobility in fibromyalgia patients. Clin Rheumatol 2012; 31(8): 1205-14.

[101] Wang C. Role of Tai Chi in the treatment of rheumatologic diseases. Curr Rheumatol Rep 2012; 14(6): 598-603.

[102] Yvonne T, Anita U, D. DB. Yoga for chronic pain management: a qualitative exploration. Scand J Caring Sci 2011; 25(3): 435-43.

[103] Johansen MY, MacDonald CS, Hansen KB et al. Effect of an Intensive Lifestyle Intervention on Glycemic Control in Patients With Type 2 Diabetes: A Randomized Clinical Trial. JAMA 2017; 318(7): 637-46.

[104] Balducci S, Zanuso S, Nicolucci A, et al. Effect of an intensive exercise intervention strategy on modifiable cardiovascular risk factors in subjects with type 2 diabetes mellitus: A randomized controlled trial: the italian diabetes and exercise study (ides). Arch Intern Med 2010; 170(20): 1794803.

[105] Praet SF, Jonkers RA, Schep G et al. Long-standing, insulin-treated type 2 diabetes patients with complications respond well to short-term resistance and interval exercise training. Eur J Endocrinol 2008; 158(2): 163-72.

[106] Fatone C, Guescini M, Balducci S et al. Two weekly sessions of combined aerobic and resistance exercise are sufficient to provide beneficial effects in subjects with Type 2 diabetes mellitus and metabolic syndrome. J Endocrinol Invest 2010; 33(7): 489-95.

[107] Lambers S, Van Laethem C, Van Acker K, Calders P. Influence of combined exercise training on indices of obesity, diabetes and cardiovascular risk in type 2 diabetes patients. Clin Rehabil 2008; 22(6): 483-92.

[108] Lee SF, Pei D, Chi MJ, Jeng C. An investigation and comparison of the effectiveness of different exercise programmes in improving glucose metabolism and pancreatic beta cell function of type 2 diabetes patients. Int J Clin Pract 2015; 69(10): 1159-70.

[109] Madsen SM, Thorup AC, Overgaard K, Jeppesen PB. High Intensity Interval Training Improves Glycaemic Control and Pancreatic $\beta$ Cell Function of Type 2 Diabetes Patients. PLoS One 2015; 10(8): e0133286. 
[110] Riva JJ, Wong JJ, Brunarski DJ et al. Consideration of chronic pain in trials to promote physical activity for diabetes: a systematic review of randomized controlled trials. PLoS One 2013; 8(8):

e71021. 